\title{
Recent highlights of spectropolarimetry applied to the magnetometry of massive stars
}

\author{
J. H. Grunhut \\ European Southern Observatory, Karl-Schwarzschild-Str. 2, D-85748 Garching, Germany \\ email: jgrunhut@eso.org
}

\begin{abstract}
Spectropolarimetry is a powerful tool used to probe fundamental properties of stars that cannot typically be measured in any other way. A new generation of high-resolution spectropolarimeters (ESPaDOnS at the Canada-France-Hawaii telescope, Narval at the Télescope Bernad Lyot, and HARPSpol at the 3.6-m ESO telescope) and dedicated observing campaigns (such as the Magnetism in Massive Stars (MiMeS) project) have led to significant improvements in both our observational and theoretical understanding of the underlying physics governing massive stars. In this article I review recent advances in the field of stellar magnetism of massive stars acquired using spectropolarimetry.
\end{abstract}

Keywords. polarization, surveys, stars: magnetic fields, stars: pre-main-sequence, stars: earlytype, stars: emission-line, Be, stars: late-type, stars: Wolf-Rayet

\section{Introduction}

As discussed by Landstreet (these proceedings), the most direct method to investigate stellar magnetism is through spectropolarimetry. In particular, obtaining circularly polarized Stokes $V$ spectra, which measures the difference between left- and right-hand circular polarization, presents the easiest way to measure the exceptionally small wavelength splitting caused by Zeeman splitting, and, is in practice, only limited by the achievable signal-to-noise ratio (SNR) of an observation. Ever since the first magnetic detection in the chemically peculiar Ap star 78 Vir (Babcock 1947), this has effectively been the primary method used to detect and diagnose magnetic fields.

The study of magnetism in massive stars $\left(M \gtrsim 8 M_{\odot}\right)$ is still a relatively new field. While the first magnetic detection in the archetype magnetic B2V star $\sigma$ Ori E goes all the way back to the pivotal work of Landstreet \& Borra (1978), only a handful of new magnetic detections have been found in the years to follow (e.g. Borra \& Landstreet 1979; Landstreet 1982; Thompson \& Landstreet 1985; Bohlender et al. 1987). In fact, most of our knowledge regarding magnetism in higher-mass stars $\left(M \gtrsim 1.5 M_{\odot}\right)$ comes from studies of the chemically peculiar A- and B-type stars. This largely reflects the fact that, unlike intermediate-mass magnetic stars that show strong and distinctive photospheric chemical peculiarities compared to non-magnetic A- and B-type stars (and therefore can be used as a proxy for magnetism), the strong, radiatively-driven outflows of more massive stars generally inhibit these chemical peculiarities, making them difficult to identify. Furthermore, the relatively weak fields of these stars coupled with relatively few spectral lines from which to directly diagnose the presence of magnetism in the optical spectra of massive stars meant that these fields remained undetected by previous generations of instrumentation.

Over the last 15 years a new generation of instruments [ESPaDOnS at the CanadaFrance-Hawaii telescope (CFHT), Narval at the Télescope Bernad Lyot (TBL), and 
HARPSpol at the ESO's 3.6-m telescope] and new techniques, such as Least-Squares Deconvolution (LSD; Donati et al. 1997), have been at the centre of studies that have significantly contributed to our understanding of magnetism in massive stars. Their large wavelength coverage (ideal for multi-line techniques) in addition to their high efficiency and their mounting on medium-sized telescopes (which allow us to reach high SNR in a reasonable amount of time for these stars), have provided us with the necessary tools to really study the magnetic properties of massive stars.

\section{Magnetism in main sequence stars}

It is well-known that magnetic fields are detected in a small population of main sequence (MS) massive stars. The majority of the magnetically detected massive stars are high-mass extensions of the chemically-peculiar intermediate-mass stars that show He peculiarities (e.g. Bohlender et al. 1987). It was only about 15 years ago that the first confirmed detections of magnetic fields in stars more massive than $\sim 10 M_{\odot}$ (earlier than B2) were made, e.g. the classical B1III pulsator $\beta$ Cep (Henrichs et al. 2000; Donati et al. 2001 ) and the young massive O7V star $\theta^{1}$ Ori C (Donati et al. 2002). In fact, to give the reader a sense of how young the field really is, prior to 2009 only 3 magnetic O-type stars were even known ( $\theta^{1}$ Ori C; the Of?p star HD 191612 (Donati et al. 2006); the evolved O9.7Ib binary star $\zeta$ Ori A (Bouret et al. 2008)), and 9 of the 13 of the most massive stars (earlier than B2) were discovered between 2005 and 2009.

The majority of what we knew about magnetism in higher-mass stars is derived from observations of intermediate-mass stars $\left(1.5<M<8 M_{\odot}\right)$. Compared to the magnetic fields found in low-mass stars $\left(M \lesssim 1.5 M_{\odot}\right)$, the incidence and characteristics of magnetic fields found in these stars are significantly different. While magnetism in low-mass stars is essentially ubiquitous, magnetic fields are only detected in 5-10\% of the population of intermediate-mass stars (e.g. Bagnulo et al. 2006). Observations suggest that the majority of intermediate-mass stars present globally-ordered, topologically simple magnetic fields, often characterized by a dipole or a low-order multipole (e.g. Aurière et al. 2007) that are effectively frozen into the star - the fields appear to be stable over timescales of at least decades and any observed variability is a consequence of rotational modulation (e.g. Silvester et al. 2012).

While strong, detectable surface magnetic fields in massive stars appear to be rather rare, they nevertheless have an important impact. Magnetic fields can confine and modulate the highly ionized radiatively driven winds of massive stars (e.g. ud-Doula \& Owocki 2002), which is likely responsible for the production of periodic high-energy X-ray emission (e.g. Gagne et al. 1997; Babel \& Montmerle 1997) and other line-profile-variability that is commonly observed in hot OB stars (e.g. Grunhut et al. 2012a,b). Furthermore, the interaction of the magnetic field with the stellar wind may enhance the shedding of angular momentum, and therefore have a significant impact on the rotation rates of OB stars (e.g. Weber \& Davis 1967; ud-Doula et al. 2008). These effects have far-reaching consequences on the evolution of massive stars, which has been shown to be strongly influenced by stellar rotation (e.g. Meynet et al. 2011). Therefore, it is necessary that the magnetic field characteristics be well understood for massive stars. To date, the largest and most productive investigation of the magnetic properties of massive stars has been the Magnetism in Massive Stars (MiMeS) Project.

The MiMeS Project was formed by an international team of recognized researchers with the goal to better understand the complex and puzzling field of magnetism of massive stars. In 2008, the MiMeS Project was awarded "Large Program" (LP) status (640 hours) by Canada and France (PI G. Wade) with ESPaDOnS from late 2008 through 
2012. This was shortly followed by additional LPs with Narval (a total of $\sim 500$ hours, PI C. Neiner) and with HARPSpol (a total of $~ 300$ hours, PI E. Alecian). In addition to these LPs, the MiMeS Project was and still is supported by numerous PI programs from such observatories as the Anglo-Australia Telescope, Chandra, Dominion Astrophysical Observatory, HST, MOST, SMARTS, and the VLT.

One aspect of the MiMeS Project includes a "Survey Component" (SC), the main goal of which was to discover newly magnetic stars and to provide critical missing information about the incidence and statistical properties of large-scale magnetic fields in a statistically-significant sample of massive stars. Over 4800 high-precision (median signal-to-noise ratio $(\mathrm{S} / \mathrm{N})$ of $\sim 800$ per pixel), high-resolution $(R \sim 68000)$, broad-band (370-1050 nm) Stokes $V$ spectra were analyzed within the context of the MiMeS Project, corresponding to approximately 550 OB stars with spectral types between B9 and O5. In order to increase the SNR and enhance our sensitivity to weak magnetic Zeeman signatures, the LSD technique, as implemented by Kochukhov et al. (2010), was used to produce mean profiles from the unpolarized light (Stokes $I$ ), circularly polarized light (Stokes $V$ ) and also from the diagnostic spectrum $(N$; which diagnoses the presence of spurious signatures resulting from instrumental or other first-order systematic effects). The detection of any signal in the LSD mean $V$ and $N$ profiles was diagnosed using a standard $\chi^{2}$ analysis (Donati et al. 1997).

Of the $\sim 550$ stars observed within the context of the MiMeS Project, approximately 65 show evidence for magnetic fields. Of these detected targets, about 30 were previously established as magnetic stars prior to the survey and are therefore ignored in the following statistics. The bulk incidence (i.e. the total number of previously-unknown magnetic stars in the sample relative to the total sample) is $7 \pm 1 \%$. Approximately 420 B-type stars in the sample were previously unknown to host magnetic fields, and of this sample, 32 stars were found to be magnetic. This translates into a magnetic incidence fraction of $7 \pm 1 \%$. Of particular interest to this conference are the most massive stars in the sample - the O-type stars. Approximately 95 O-type stars in the survey were previously unknown to host magnetic fields, and 6 were found to be magnetic, for an incidence of $6 \pm 1 \%$. The MiMeS Project has carried out that largest and most sensitive investigation of the magnetic properties of O-type stars, and in the end has now tripled the number of previously known magnetic O-type stars.

The detected magnetic stars (e.g. Grunhut et al. 2012a,b; Wade et al. 2012a) exhibit periodic variability of their Stokes $V$ profiles (with periods in the range of $0.5 \mathrm{~d}$ to many years), as a result of the rotational modulation of the projected magnetic field. These detections were systematically found to present organized magnetic fields with important dipole components. The surface polar field strengths range from several hundred $\mathrm{G}$ up to $20 \mathrm{kG}$. In general, the magnetic characteristics of detected B-type stars and O-type stars are very similar. Of particular note, the MiMeS Project is responsible for discovering the most rapidly-rotating, magnetic, early-type stars (HR 7355 (Oksala et al. 2010; Rivinius et al. 2010, 2013); HR 5907 (Grunhut et al. 2012a)) and the most strongly magnetic O-type star (NGC 1624-2 (Wade et al. 2012b)) known to date.

Based on the statistics learned from the MiMeS Project, it can be concluded that the incidences and characteristics of large-scale magnetic fields in B- and O-type stars are essentially the same. The physical and statistical properties of their fields are qualitatively identical to those of intermediate-mass stars with spectral types between F0 to A0 on the MS. The MiMeS survey therefore establishes that the basic physical characteristics of magnetism in stars with radiative envelopes remains unchanged across more than 1.5 decades of stellar mass, from mid-F to early O-type stars $\left(M \sim 1.5 \mathrm{M}_{\odot}\right.$ to $\left.\sim 50 \mathrm{M}_{\odot}\right)$. 
Other than providing critical missing information regarding the statistical properties of magnetic fields in massive stars, another goal of the survey was to investigate the connection between magnetism and observed phenomena. Listed here are some of these results.

The MiMeS Project has observed all of the known Galactic stars of the Of?p class, and detected or confirmed the presence of a magnetic field in each of these stars (see e.g. Wade et al. $2012 \mathrm{~b}$, and references therein). The Of?p classification pertains to O-type stars that exhibit CIII $\lambda 4650$ in emission with a strength comparable to the neighbouring NiII lines (Walborn 1972; Walborn et al. 2010). It can therefore be concluded that the Of?p stars represent a magnetic class of O-type stars, and the peculiar spectral properties are likely a consequence of the interaction of their winds with the magnetic fields (e.g. Sundqvist et al. 2012). This is the first established magnetic class of O-type stars (similar to the chemically-peculiar Ap/Bp stars), and as there are now 5 known extra-Galactic Of?p stars (Walborn et al. 2010; Massey et al. 2014), this therefore implies the identification of the first magnetic stars outside of the Galaxy.

About 100 pulsating $\beta$ Cep and slowly-pulsating B-type (SPB) stars were observed as part of the MiMeS SC. It has recently been suggest (Hubrig et al. 2006, 2009) that there is a high magnetic incbidence fraction $(\sim 50 \%)$ among these stars, which has far-reaching consequences regarding the connection between pulsations and magnetism. However, the MiMeS SC results suggest a significantly different result with a magnetic incidence fraction of $10 \pm 3 \%$. This value is fully consistent with the larger sample of O- and Btype stars and suggests that pulsating B-type stars are not more likely to be magnetic than non-pulsating B-type stars. Furthermore, a monitored investigation of a sample of claimed magnetic detections with characterized magnetic models by Hubrig et al. (2011) have also been shown to be inconsistent with the MiMeS data (Shultz et al. 2012). It can therefore be concluded that there is no connection between magnetism and pulsations in B-type stars.

In a sample of about 90 classical Be stars, MiMeS has failed to unambiguously detect a magnetic field in any of these stars. The sample of classical Be stars were identified according to the classification of Porter \& Rivinius (2003) - as early-type emission line stars with Keplerian disks - to distinguish these stars from other emission line stars whose emission is of a different origin (e.g. accretion disks from Herbig stars). Be stars are also known to be rapidly-rotating (e.g. Rivinius et al. 2013), yet despite this fact, MiMeS has achieved a magnetic sensitivity similar to that of the larger sample and has successfully detected fields in other rapidly-rotating stars (e.g. Oksala et al. 2010; Grunhut et al. 2012a) and therefore expected to detect fields in $\sim 10$ stars. The majority of the Be sample was chosen to exclude stars whose optical spectra were dominated by emission lines, but most still showed significant emission. However, the lack of detections is not a result of this emission as magnetic fields have been detected in other emission-line stars (e.g. Herbig Be stars or Of?p stars). Hence it can be concluded that the lack of detected magnetic classical Be stars, indicates that decretion Keplerian Be disks are not of magnetic origin (Neiner et al. 2012).

\section{Magnetism in pre-main sequence stars}

The pre-main sequence (PMS) phase of evolution is defined as the period of stellar formation after the proto-stellar phase, but before the MS phase where a star is undergoing core-hydrogen burning. At the beginning of the PMS phase the star is on the birth-line - the locus of points in the HR diagram where the star is, for the first time, observable at optical wavelengths after shedding the majority of its proto-stellar envelope and is 
no longer undergoing significant accretion (Stahler 1983). At this point, nuclear-burning has not yet started in the star's core and slow gravitational collapse is the main source of energy. Just before the end of the PMS phase, nuclear reactions begin, contributing more and more energy to the luminosity of the star. As the star evolves closer to the zero age main sequence (ZAMS), gravitational contraction slows, eventually coming to a stop.

As previously discussed, main sequence, magnetic higher-mass stars all show stable large-scale fields, at least over the lifetime observations. The leading hypothesis suggests that these magnetic fields are fossil fields - the frozen remnants of the Galactic field accumulated and possibly enhanced by a dynamo field generated in an earlier phase of evolution (e.g. Cowling 1945; Mestel 2001; Moss 2001). This has been supported by numerical and analytical studies that find that surviving fields can relax into configurations exhibiting long-term stability (Braithwaite 2009; Duez \& Mathis 2010) and can approximately explain the field topology and other general characteristics of magnetism in higher-mass stars. However, prior to about 2005, the global magnetic properties of HAeBe stars were unknown, and therefore direct evidence linking the magnetic properties of MS stars with PMS stars was completely lacking.

To address this issues, a high-resolution spectropolarimetric survey of 128 Herbig Ae/Be (HAeBe) stars (Herbig 1960; Vieira et al. 2003) was carried out using ESPaDOnS and Narval. A sample of field HAeBe stars were selected from the catalogue of The et al. (1994) and Vieira et al. (2003), while an additional sample were selected from members of the following young clusters: NGC 2244 (Park \& Sung 2002), NGC 2264 (Sung et al. 1997), and NGC 6611 (de Winter et al. 1997). LSD was used to increase the sensitivity to detect weak Zeeman signatures. This survey obtained magnetic detections in 8 stars (e.g. Wade et al. 2005; Alecian et al. 2008b) and found a magnetic incidence fraction of $\sim 6 \%$, similar to the incidence that is found for intermediate-mass MS stars and now MS massive stars. Spectropolarimetric monitoring of many of the detected stars was also carried out in order to characterize the strength and structure of their surface fields. The results show that the magnetic properties of HAeBe star are similar to what is found on the MS - the fields are mainly dipolar, are strong (surface field strengths of $300 \mathrm{G}$ to $4 \mathrm{kG}$ ), and stable over many years (e.g. Alecian et al. 2008a, 2009). This survey has therefore established a direct link between the magnetic properties of stars found on the PMS and on the MS. It can therefore be concluded that the magnetic properties of A/B stars must have been shaped before the HAeBe phase of stellar evolution (Alecian et al. 2013).

\section{Magnetism in evolved massive stars}

As a massive star completes its MS evolution, its envelope rapidly grows while its surface temperature cools until it eventually forms a cool supergiant. Characterized by a helium-burning core and a deep convective hydrogen-burning envelope, the internal structure of a supergiant is in strong contrast to its MS OB progenitor, which is characterized by a convective hydrogen-burning core and a radiative envelope. The most massive stars (initial mass $>25 M_{\odot}$ ) go through a phase of extreme mass-loss and eject their envelope in the Wolf-Rayet (WR) phase before ending their lives in a dramatic Type Ib supernova explosion, while the less massive stars (initial mass between about 8 and $25 M_{\odot}$ ) evolve into red supergiants that explode as Type II supernovae. Ultimately, the end product of a massive star is a neutron star or black hole (e.g. Crowther et al. 1995; Eldridge 2008).

It is well known that magnetic fields exist in at least some fraction of massive stars and that magnetic flux conservation of the fields found in MS A- and B-type stars is in 
relative agreement with the fluxes observed in their ultimate descendants: white dwarfs and neutron stars (e.g. Tout et al. 2004; Ferrario \& Wickramasinghe 2006; Walder et al. 2012). It therefore is unsurprising to expect that at least some fraction of stars found in an intermediate phase of evolution should be magnetic.

\subsection{Wolf-Rayet stars}

While it has only recently been established by the MiMeS collaboration, and now confirmed by the B-fields in OB stars (BOB) collaboration (see e.g. Morel et al., these proceedings), that magnetic fields are systematically detected in about $5-10 \%$ of stars with radiative envelopes, WR stars, which are the direct descendants of the most massive OB stars, are known to show evidence of corotating interaction regions (CIRs), which some have postulated to be the result of magnetic fields (Harries 2000). Motivated by this and the previously discussed matter, de la Chevrotière et al. (2013, 2014) (as part of the MiMeS collaboration) have recently attempted to constrain the magnetic field characteristics of these stars.

However, unlike the previously discussed results for MS and PMS stars, the diagnosis and detection of magnetic fields in WR stars is complicated by the fact that the majority of the visible optical spectral lines are in emission, a consequence of their formation in a strong stellar wind. Not only does this make the application of multi-line techniques extremely difficult to be useful, but this also has the potential to fundamentally change the Stokes $V$ profile shape relative to the signatures that are formed in the stellar photosphere - the typical large-scale field in higher-mass stars is generally well-described by a dipole; however, in the presence of a strong stellar wind, or at large distances from the photosphere (where the wind energy density is greater than the magnetic energy density), the field lines are stretched open and better resemble a split monopole (udDoula \& Owocki 2002) - and therefore makes characterization of these lines challenging. Gayley \& Ignace (2010) have investigated this issue and have developed a model of the expected shape of a Zeeman signature formed in stellar wind. As expected, the shape of the Zeeman signature formed in an outflowing wind with an underlying split monopole configuration is considerably different from that of a Zeeman signature formed in a hydrostatic atmosphere with an underlying mainly-dipole configuration: the latter can usually be well-described by an $s$-shaped profile, while the former reflects a heartbeat shape to its profile (see Fig. 3 of Gayley \& Ignace 2010 for an example).

Taking all this into consideration, de la Chevrotière et al. (2013, 2014) have carried out the most sensitive and detailed search for the presence of large-scale magnetic fields in 12 WR-stars. None of their observations resulted in the unambiguous detection of any Zeeman signature in their data. However, using Bayesian inference methods, they were able to derive upper limits for these non-detections and, in some stars, find marginal evidence favouring a non-zero magnetic model. They find an average upper limit of the allowed field strength of $\sim 500 \mathrm{G}$ in the wind is consistent with the non-detections in their sample and that 3 of the 12 stars show marginal evidence for magnetic fields with likely field strengths of $\sim 100-200 \mathrm{G}$, or not exceeding $\sim 2 \mathrm{kG}$ at their most upper limits permitted by their Bayesian assessment. If these marginal detections are taken at face value, then this would imply a considerably higher magnetic incidence fraction $(\sim 25 \%)$ among WR stars than their progenitors (the OB stars with a $\sim 7 \%$ magnetic incident rate). However, robust conclusions cannot be derived from such small number statistics, as also pointed out by the authors. 


\section{Magnetism in cool supergiants}

Active late-type supergiants are luminous in X-rays and show emission in chromospheric ultraviolet (UV) Si IV lines, with some stars showing evidence of flaring phenomena usually associated with the presence of a corona and magnetic reconnection generated via dynamo fields (e.g. Tarasova 2002; Ayres 2005). Additionally, a class of 'non-coronal' or inactive supergiants - that exhibit weak or no X-ray emission and have UV spectra containing mostly low-temperature or relatively weaker flux chromospheric lines compared to 'normal' supergiants - are known to exist, and can be explained by the presence of magnetic loops submerged within their thick extended chromospheres (Ayres 2005). Furthermore, observations of Betelgeuse have revealed evidence for an extended chromosphere (Carpenter \& Robinson 1997) and surface brightness fluctuations that can be described by giant convective cells (e.g. Chiavassa et al. 2010, and references therein).

These observational phenomena have hinted that significant magnetic fields should be present in supergiants. The first confirmation of this was the detection of Zeeman signatures found in the mean LSD profiles obtained with high-resolution spectropolarimeters corresponding to a magnetic field in Betelgeuse (Aurière et al. 2010) and the systematic detection of magnetic fields in a larger population of supergiants (Grunhut et al. 2010). The original sample presented by Grunhut et al. (2010) has more than doubled in size and now contains about 70 stars. New statistics derived from this sample indicate that approximately $\gtrsim 30-40 \%$ of supergiant stars are found to have detected Zeeman signatures in their mean LSD profiles (an example is provided in Fig. 1). An HR-diagram showing the position of these detected stars is presented in Fig. 1. The detected fields have globally weak surface-averaged magnetic fields, as inferred from their weak longitudinal magnetic field measurements on the order of $\sim \mathrm{G}$ (compared to the 100-1000s of $\mathrm{G}$ in their $\mathrm{OB}$ progenitors). The characteristics of these profiles suggest that many of these stars host topologically complex fields that significantly depart from the largescale dipoles typically found in their OB progenitors. If we just include stars that are cooler than the granulation boundary that suggests the onset of a significant convective envelope (Gray 1989) (as shown in Fig. 1), then the incidence rate is somewhat higher at $\sim 50-60 \%$. For stars hotter than the granulation boundary, the incidence rate drops significantly to $\sim 10 \%$. Based on these results we can make some basic conclusions: the significantly higher incidence fraction of magnetic fields among cool supergiants suggests these stars cannot be the descendants of the magnetic OB stars. The higher incidence fraction of fields among stars with deep convective envelopes suggest that these fields are likely drive by dynamo mechanisms (e.g. Parker 1955); however, the extremely slow rotation of the coolest supergiants (such as Betelgeuse) would argue for an exotic dynamo model for at least some of these stars, as they are rotating too slow for the solar-like $\alpha-\omega$ dynamo models to be efficient. Numerical models of Dorch (2004) have already shown that magnetic fields can be generated from large convective cells.

\section{Conclusions}

It is now well-established that large-scale magnetic fields, detected from the induced circularly polarized signatures reflecting Zeeman splitting in the mean line profiles of high-resolution spectra, are detected in at least some fraction of stars at every phase of stellar evolution of massive stars - from the pre-MS all the way to the latest stages of evolution before the star violently explodes. The similar statistics and magnetic properties of massive stars during the pre-MS and MS phases suggests that the physics of magnetism in stellar radiative zones remains unchanged over millions of years of evolution, and 

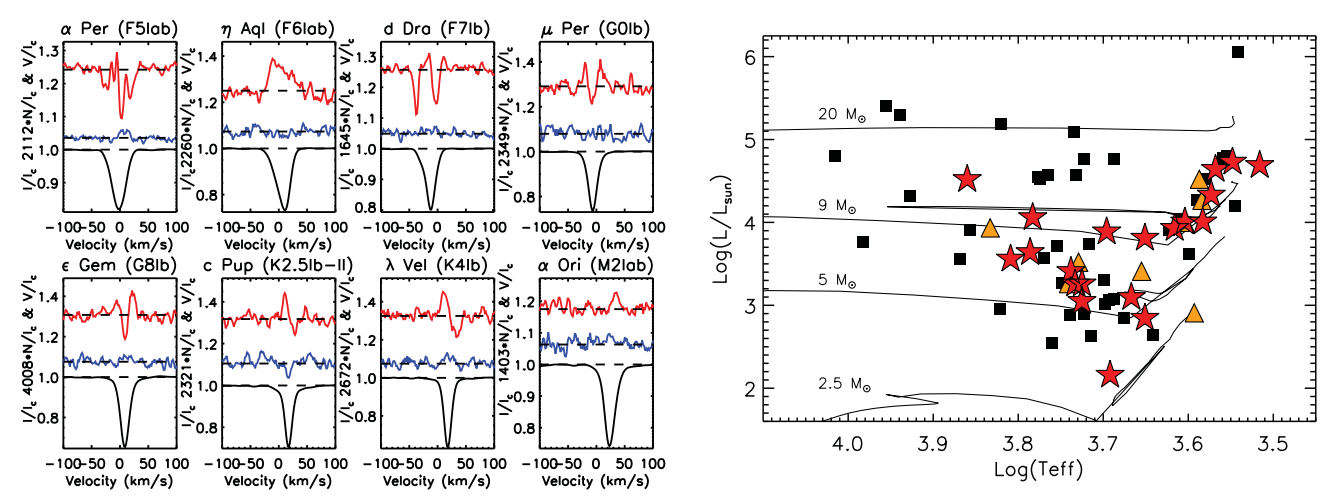

Figure 1. Left: Example mean Stokes $V$ (top), diagnostic null (middle) and Stokes $I$ line profiles obtained from cool supergiants. The profiles in the upper panels are likely a consequence of topologically complex surface magnetic fields in these stars. Right: HR diagram showing the position of all supergiants observed as part of a survey of cool supergiants. Evolutionary tracks (sold lines) are also included. Black squares correspond to stars with non detection of a Zeeman signature, orange triangles reflect stars with a possibly detected Zeeman signature, and red stars represent stars with an unambiguously detected Zeeman signature.

that the detected fields are formed and fossilized in the star prior to the pre-MS phase. With only a small number of WR stars investigated in any detail and no unambiguous detection of a field in these stars, no conclusions can be drawn about their potential relationship to possible magnetic OB star progenitors. Lastly, as the magnetic incidence fraction of magnetism in cool supergiants greatly exceeds similar measurements in MS massive stars, it stands to reason that the majority of supergiants are not the descendants of MS magnetic stars. This suggests that the detected fields are not fossil remnants of an earlier phase of evolution and are therefore likely generated contemporaneously via some dynamo process.

\section{References}

Alecian, E., Catala, C., Wade, G. A., et al. 2008a, MNRAS 385, 391

Alecian, E., Wade, G., Catala, C., et al. 2008b, A $\mathscr{E} A$ 481, L99

Alecian, E., Wade, G. A., Catala, C., et al. 2009, MNRAS 400, 354

Alecian, E., Wade, G. A., Catala, C., et al. 2013, MNRAS 429, 1001

Aurière, M., Donati, J.-F., Konstantinova-Antova, R., et al. 2010, A $\& A$ 516, L2

Aurière, M., Wade, G. A., Silvester, J., et al. 2007, A\&SA 475, 1053

Ayres, T. R. 2005, ApJ 618, 493

Babcock, H. W. 1947, ApJ 105, 105

Babel, J. \& Montmerle, T. 1997, A\&A 323, 121

Bagnulo, S., Landstreet, J. D., Mason, E., et al. 2006, A\&A 450, 777

Bohlender, D., Landstreet, J., Brown, D., \& Thompson, I. 1987, ApJ 323, 325

Borra, E. F. \& Landstreet, J. D. 1979, ApJ 228, 809

Bouret, J.-C., Donati, J.-F., Martins, F., et al. 2008, MNRAS 389, 75

Braithwaite, J. 2009, MNRAS 397, 763

Carpenter, K. G. \& Robinson, R. D. 1997, ApJ 479, 970

Chiavassa, A., Haubois, X., Young, J. S., et al. 2010, A\& A 515, A12

Cowling, T. G. 1945, MNRAS 105, 166

Crowther, P. A., Hillier, D. J., \& Smith, L. J. 1995, AछA 293, 172

de la Chevrotière, A., St-Louis, N., \& Moffat, A. F. J., the MiMeS Collaboration 2013, ApJ 764, 171 
de la Chevrotière, A., St-Louis, N., \& Moffat, A. F. J., the MiMeS Collaboration 2014, ApJ 781, 73

de Winter, D., Koulis, C., The, P. S., et al. 1997, A\&AS 121, 223

Donati, J.-F., Babel, J., Harries, T., et al. 2002, MNRAS 333, 55

Donati, J.-F., Howarth, I., Bouret, J.-C., et al. 2006, MNRAS 365, L6

Donati, J.-F., Semel, M., Carter, B., Rees, D., \& Collier Cameron, A. 1997, MNRAS 291, 658

Donati, J.-F., Wade, G., Babel, J., et al. 2001, MNRAS 326, 1265

Dorch, S. B. F. 2004, $A \mathscr{E} A 423,1101$

Duez, V. \& Mathis, S. 2010, A\&A 517, A58

Eldridge, J. J. 2008, Royal Society of London Philosophical Transactions Series A 366, 4441

Ferrario, L. \& Wickramasinghe, D. 2006, MNRAS 367, 1323

Gagne, M., Caillault, J.-P., Stauffer, J. R., \& Linsky, J. L. 1997, ApJL 478, L87

Gayley, K. G. \& Ignace, R. 2010, ApJ 708, 615

Gray, D. F. 1989, PASP 101, 832

Grunhut, J. H., Rivinius, T., Wade, G. A., et al. 2012a, MNRAS 419, 1610

Grunhut, J. H., Wade, G. A., Hanes, D. A., \& Alecian, E. 2010, MNRAS 408, 2290

Grunhut, J. H., Wade, G. A., Sundqvist, J. O., et al. 2012b, MNRAS 426, 2208

Harries, T. J. 2000, MNRAS 315, 722

Henrichs, H. F., de Jong, J. A., Donati, D.-F., et al. 2000, in Y. V. Glagolevskij \& I. I. Romanyuk (eds.), Magnetic Fields of Chemically Peculiar and Related Stars, pp 57-60

Herbig, G. H. 1960, ApJS 4, 337

Hubrig, S., Briquet, M., De Cat, P., et al. 2009, Astronomische Nachrichten 330, 317

Hubrig, S., Briquet, M., Schöller, M., et al. 2006, MNRAS 369, L61

Hubrig, S., Ilyin, I., Schöller, M., et al. 2011, ApJL 726, L5

Hussain, G. A. J. \& Alecian, E. 2014, in IAU Symposium, Vol. 302 of IAU Symposium, pp 25-37

Kochukhov, O., Makaganiuk, V., \& Piskunov, N. 2010, A\&A 524, A5

Landstreet, J. \& Borra, E. 1978, ApJL 224, L5

Landstreet, J. D. 1982, ApJ 258, 639

Massey, P., Neugent, K. F., Morrell, N., \& Hillier, D. J. 2014, ApJ 788, 83

Mestel, L. 2001, in G. Mathys, S. Solanki, \& D. Wickramasinghe (eds.), Magnetic Fields Across the Hertzsprung-Russell Diagram, Vol. 248 of Astronomical Society of the Pacific Conference Series, p. 3

Meynet, G., Eggenberger, P., \& Maeder, A. 2011, A\&A 525, L11

Moss, D. 2001, in G. Mathys, S. Solanki, \& D. Wickramasinghe (eds.), Magnetic Fields Across the Hertzsprung-Russell Diagram, Vol. 248 of Astronomical Society of the Pacific Conference Series, p. 305

Neiner, C., Grunhut, J. H., Petit, V., et al. 2012, MNRAS 426, 2738

Oksala, M., Wade, G., Marcolino, W., et al. 2010, MNRAS 405, L51

Park, B.-G. \& Sung, H. 2002, AJ 123, 892

Parker, E. N. 1955, ApJ 122, 293

Porter, J. M. \& Rivinius, T. 2003, PASP 115, 1153

Rivinius, T., Carciofi, A. C., \& Martayan, C. 2013, A $\& A$ Rev. 21, 69

Rivinius, T., Szeifert, T., Barrera, L., et al. 2010, MNRAS 405, L46

Shultz, M., Wade, G. A., Grunhut, J., et al. 2012, ApJ 750, 2

Silvester, J., Wade, G. A., Kochukhov, O., et al. 2012, MNRAS 426, 1003

Stahler, S. W. 1983, ApJ 274, 822

Sundqvist, J., ud-Doula, A., Owocki, S., et al. 2012, MNRAS p. L433

Sung, H., Bessell, M. S., \& Lee, S.-W. 1997, AJ 114, 2644

Tarasova, T. N. 2002, Astronomy Reports 46, 474

The, P. S., de Winter, D., \& Perez, M. R. 1994, A\&AS 104, 315

Thompson, I. B. \& Landstreet, J. D. 1985, ApJL 289, L9

Tout, C. A., Wickramasinghe, D. T., \& Ferrario, L. 2004, MNRAS 355, L13

ud-Doula, A. \& Owocki, S. 2002, ApJ 576, 413

ud-Doula, A., Owocki, S., \& Townsend, R. 2008, MNRAS 385, 97 
Vieira, S. L. A., Corradi, W. J. B., Alencar, S. H. P., et al. 2003, AJ 126, 2971

Wade, G., Grunhut, J., Gräfener, G., et al. 2012a, MNRAS 419, 2459

Wade, G. A., Drouin, D., Bagnulo, S., et al. 2005, A\&3A 442, L31

Wade, G. A., Maíz Apellániz, J., Martins, F., et al. 2012b, MNRAS 425, 1278

Walborn, N. R. 1972, AJ 77, 312

Walborn, N. R., Sota, A., Maíz Apellániz, J., et al. 2010, ApJL 711, L143

Walder, R., Folini, D., \& Meynet, G. 2012, Space Science Reviews 166, 145

Weber, E. \& Davis, Jr., L. 1967, ApJ 148, 217

\section{Discussion}

KHALACK: Could you please comment on the possibility of detecting magnetic fields in the Horizontal-Branch (HB) stars?

Grunhut: There are currently no plans to observe HB stars. As the HB stars are typically found (and also identified) in Globular clusters, these stars tend to be too faint to acquire high-resolution spectropolarimetry with the current generation of instruments and the telescopes they are mounted on. Perhaps this is possible with low-resolution instruments that are mounted on larger telescopes (such as FORS on the VLT).

MAEDER: Are you planning to also observe magnetic fields in evolutionary stages earlier that the Herbig Ae/Be phase, such as stars that live in the Hayashi or FU Orionis phase?

Grunhut: Evelyne Alecian and Gaitee Hussain are already leading a project that aims to observe the earliest phases of intermediate-mass evolution that is permitted by optical spectropolarimetric studies (Hussain \& Alecian 2014). At earlier evolutionary phases the star is still enshrouded in its cocoon and therefore infrared spectropolarimeters are required to observe stars at these phases. Therefore, one of the primary goals for the next generation of spectropolarimeters (e.g. SPIRou at the CFHT, CRIRES+ at the VLT) is to probe the magnetic properties of these young stars in the early stages of their formation.

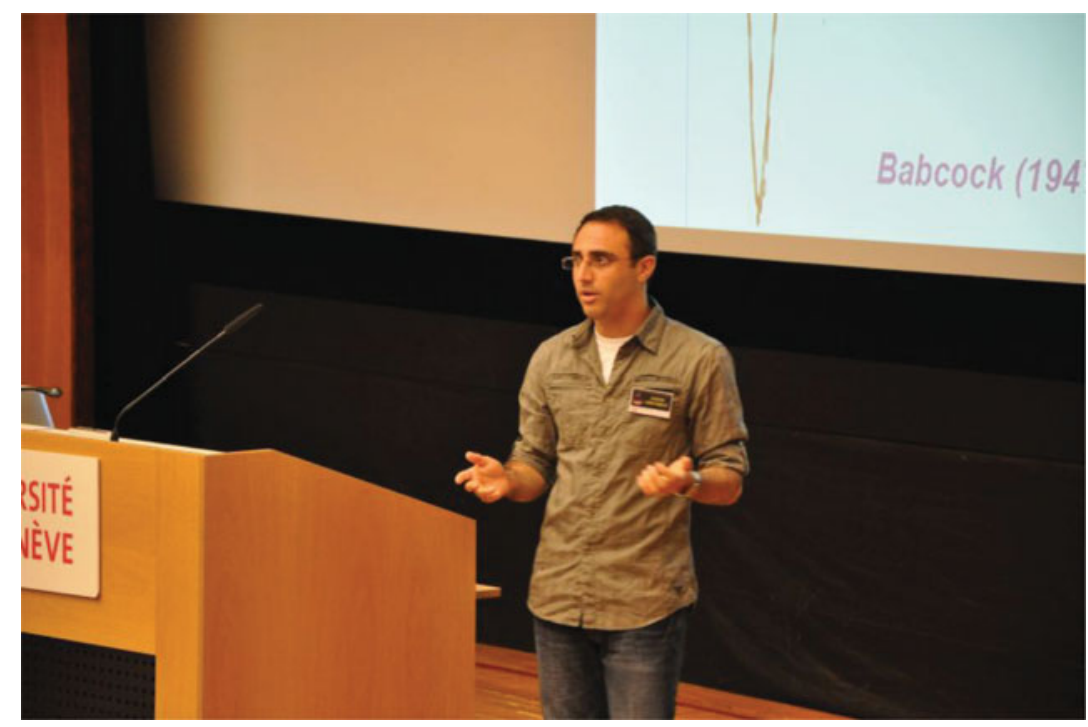

Jason Grunhut 